\title{
Références bibliographiques du dossier « La recherche en éducation »
}

Bernadette Plumelle

\section{(2) OpenEdition \\ 12 Journals}

Édition électronique

URL : https://journals.openedition.org/ries/10131

DOI : 10.4000/ries. 10131

ISSN : 2261-4265

Éditeur

France Education international

\section{Édition imprimée}

Date de publication : 1 décembre 2020

Pagination : 153-164

ISBN : 978-2-85420-628-9

ISSN : 1254-4590

Référence électronique

Bernadette Plumelle, "Références bibliographiques du dossier "La recherche en éducation » ", Revue internationale d'éducation de Sèvres [En ligne], 85 | décembre 2020, mis en ligne le 01 décembre 2020, consulté le 07 décembre 2022. URL : http://journals.openedition.org/ries/10131 ; DOI : https://doi.org/ 10.4000/ries.10131 


\title{
Références bibliographiques du dossier "La recherche en éducation "
}

\section{Bernadette Plumelle}

France Éducation International

La recherche en éducation occupe actuellement une place de premier plan dans le paysage éducatif, parce que les rapports qu'elle entretient avec le monde politique et scientifique se sont transformés et renforcés. Dans tous les pays, la tendance est à utiliser les résultats de la recherche pour améliorer l'éducation et multiplier les partenariats entre recherche et pratique. La littérature sur ce sujet est trop abondante pour restituer les débats dans chaque région du monde. Les références sélectionnées pour cette bibliographie, très majoritairement en langue française, sont récentes pour présenter un état des débats actuels.

Cette sélection rassemble, dans une première partie, quelques références sur les sciences de l'éducation en France, qui font le bilan des cinquante ans de ce champ disciplinaire et en dessinent les perspectives. La section centrale questionne les méthodes, enjeux et diffusion de la recherche en éducation. Les deux parties suivantes analysent les relations complexes de la recherche en éducation avec les politiques éducatives et avec les pratiques des enseignants. Enfin, la dernière partie rassemble des publications sur des questions débattues: l'enseignement par les preuves et la place des neurosciences dans l'éducation.

Les titres proposés dans cette sélection complètent celles données par les auteurs des articles du dossier. Les résumés sont, pour l'essentiel, ceux des éditeurs et des revues.

Bibliographie arrêtée le 5 décembre 2020.

\section{LES SCIENCES DE L'ÉDUCATION : UN CHAMP DISCIPLINAIRE RÉCENT}

\begin{abstract}
ALBERO Brigitte, "Les sciences de l'éducation au XXI siècle: vers une consolidation disciplinaire de la section?", Les Dossiers des sciences de l'éducation, 2019, $n^{\circ} 41$, p. 21-42 [en ligne]
\end{abstract}

L'article propose de mettre en évidence certaines caractéristiques des sciences de l'éducation (SE) : 1) une différenciation constitutive entre champ de recherche et champ de pratiques; 2) la coexistence d'orientations pluri-, inter- et transdisciplinaires; 3 ) une triple finalité (épistémique, pragmatique, axiologique) qui pourrait constituer une identité disciplinaire; 4) une tension structurante entre épistémè et praxis qui explique les voisinages et les dissemblances entre recherche à propos du phénomène éducatif et action éducative. Enfin, l'auteure questionne l'actualité et le devenir des SE en relation avec les autres sciences humaines et sociales. https://journals.openedition.org/dse/3265 
ALTET Marguerite, " 50 ans de sciences de l'éducation: témoignages et perspectives", Les Dossiers des sciences de l'éducation, 2018, $n^{\circ}$ 40, p. 115-127 [en ligne]

L'auteure reprend les points de vue de quinze grandes figures représentatives de l'histoire des sciences de l'éducation (SE) en France au cours des cinquante dernières années. Leur ont été demandés des textes courts sur une controverse importante à leurs yeux dans l'histoire des sciences de l'éducation. L'article développe les perspectives qu'ils entrevoient pour le futur de la discipline avec 1) les risques à surmonter autour de l'hégémonie des neurosciences, de la place des disciplines sœurs, et de l'utilité sociale de la discipline SE; et 2) un avenir prometteur sous une figure composite et équilibrée entre développement d'un champ scientifique pluridisciplinaire et traitement d'enjeux professionnels et sociaux.

https://journals.openedition.org/dse/3013

ALTET Marguerite, MARCEL Jean-François (coordinateurs), "Les sciences de l'éducation en France: positionnement, tensions, avancées ", Les Sciences de l'éducation pour l'ère nouvelle, 2019, vol. 52, tome 1, 128 p.; tome 2, 152 p. [disponible sur le portail Cairn]

La discipline des sciences de l'éducation a célébré ses 50 ans en 2017, donnant lieu à des manifestations scientifiques, sujet de deux numéros de la revue. L'introduction du numéro 1 présente le projet éditorial. L'analyse historique de la discipline et les controverses autour d'objets de recherche montrent l'évolution des questions vives, des problématiques, des concepts et témoignent des avancées, des ouvertures et des renoncements de ces cinquante dernières années en France.

CHARLOT Bernard, "La recherche en éducation entre savoirs, politiques et pratiques: spécificité et défis d'un champ de savoir ", Recherches \& éducations, 2008, $n^{o} 1, p .155-174$ [en ligne]

Existe-t-il un champ de savoirs spécifique, nommé «éducation » ou "sciences de l'éducation ", ou bien l'éducation est-elle simplement un domaine pratique et politique sur lequel diverses sciences humaines produisent des recherches? Sur la base de sa double culture française et brésilienne, l'auteur aborde cette question par trois approches successives: ce que les chercheurs eux-mêmes pensent de cette question, les types de discours actuellement produits sur l'éducation et ce qu'est, ou pourrait être, la spécificité d'une discipline nommée Éducation. https://journals.openedition.org/rechercheseducations/455

ÉTIENNE Richard, DUPRIEZ Vincent, MAULINI Olivier, TARDIF Maurice (coordinateurs), "La recherche francophone en éducation: réseaux, échanges, publications ", Les Dossiers des sciences de l'éducation, 2019, $n^{\circ}$ 41, p. 7-154 [en ligne]

Ce numéro offre une mise en perspective de la recherche francophone dans la discipline des sciences de l'éducation et de la formation. Il comprend une première partie consacrée au développement de la recherche en éducation, suivie de trois articles qui étudient la question de l'usage éventuel par les professionnels des apports de la recherche en éducation dans trois pays (Québec, Suisse romande, Belgique). https://journals.openedition.org/dse/3216 
GUY Daniel, DUPONT Pascal, FABRE Isabelle (coordinateurs), "Mêlées et démêlés, 50 ans de recherches en sciences de l'éducation. Dossier", Les Dossiers des sciences de l'éducation, 2018, $n^{\circ}$ 40, p. 7-127 [en ligne]

Les sciences de l'éducation atteignent en 2017 le seuil symbolique des 50 ans d'existence. Ces années de travaux, de colloques, de publications ont permis à la discipline d'asseoir sa légitimité et ont produit une histoire, faite de débats, d'avancées scientifiques, d'évolutions ou de bifurcations. Dans le prolongement du colloque du même nom (Toulouse, 2017), ce numéro revient sur les controverses qui ont jalonné cette période et font encore aujourd'hui son actualité. https://journals.openedition.org/dse/2539

HOFSTETTER Rita, SCHNEUWLY Bernard, "Sciences de l'éducation", in VAN ZANTEN Agnès, RAYOU Patrick (sous la direction de), Dictionnaire de l'éducation, Paris: PUF, 2017 (2édition), p. 778-784

Le champ disciplinaire des «sciences de l'éducation » existe aujourd'hui dans la plupart des régions du monde, avec des configurations et des désignations variées. Les auteurs retracent l'émergence de ce nouveau savoir, les premières institutionnalisations académiques puis, avec l'« explosion scolaire » de l'après-guerre, le développement des sciences de l'éducation au niveau national, avec une professionnalisation de la recherche et la constitution de réseaux qui favorisent la construction et la diffusion des connaissances scientifiques au niveau national et international.

MABILON-BONFILS Béatrice, DELORY-MOMBERGER Christine (sous la direction de), À quoi servent les sciences de l'éducation?, Paris: ESF, 2019, 228 p.

La question du lien entre savoirs savants, politiques publiques, expertises et pratiques sociales se pose aux sciences de l'éducation comme à toutes les sciences humaines et sociales. L'ouvrage interroge la situation singulière des sciences de l'éducation en tant que sciences relativement récentes dans le champ scientifique et social. Après une partie réservée aux débats taxinomiques, la deuxième partie ouvre des questionnements autour des problématiques d'apprentissage, de didactiques et de professionnalisation. La troisième partie s'intéresse aux élargissements et à la diversification de la discipline.

\section{LA RECHERCHE EN ÉDUCATION : MÉTHODES, ENJEUX ET DIFFUSION}

BROUSSAL Dominique, CHALIES Sébastien, LEBLANC Serge (coordinateurs), "Innovation - Changement: enjeux et méthodes pour la recherche en éducation. Dossier", Éducation et socialisation. Les cahiers du CERFEE, janvier 2020, $n^{\circ}$ 55, n.p. [en ligne]

L'innovation pédagogique et le changement constituent des objets bien inscrits dans le champ des recherches en sciences de l'éducation et de la formation. Dans de nombreux cas, les processus d'éducation ou de formation visent des changements individuels ou collectifs. Et la question de la transformation des pratiques et celle des changements qu'elle requiert ou entraîne n'est pas étrangère aux sciences de l'éducation. L'innovation renvoie à la question des méthodes tout comme à la capacité que les enseignants ont de trouver des solutions inédites à des difficultés d'apprentissage. https://journals.openedition.org/edso/8716 
CHARLOT Bernard, "Les recherches en éducation et formation: formes et enjeux", in MABILON-BONFILS Béatrice, DELORY-MOMBERGER Christine (sous la direction de), À quoi servent les sciences de l'éducation? Paris: ESF, 2019, p. 111-121

Il existe une grande diversité des formes de recherche en éducation qui varient avec les références disciplinaires, les objets, les démarches et les enjeux. L'auteur identifie tout d'abord des blocs de recherche pour opérer un premier regroupement. Il réfléchit ensuite aux raisons de cette multiplicité et à ses conséquences. Enfin, il analyse les dominantes de la recherche en éducation dans la société contemporaine.

COLLARD-FORTIN Ugo, BARON Marie-Pierre, BRUYÈRE Marie-Hélène, "Les revues scientifiques en science de l'éducation: comment être singulier dans une offre plurielle? ", Revue hybride de l'éducation, automne 2018, vol. 2, n 2, p. 103-109 [en ligne]

Les acteurs de l'édition scientifique et les organisations qu'ils représentent doivent amorcer une réflexion sur les meilleurs moyens de diffusion à l'époque du numérique. Dans cet article, les auteurs se penchent sur différents enjeux du monde de l'éducation, ceux relatifs au transfert des connaissances issues de la recherche comme ceux liés aux impératifs de la publication scientifique. https://bit.ly/3otLVuy

DE KETELE Jean-Marie, "La recherche scientifique en éducation: quels critères de qualité? ", Education Sciences \& Society, 2010, p. 21-39 [en ligne]

À quels critères soumettre la qualité du processus des recherches scientifiques dont l'objet est l'éducation ou la formation? Comment, dans ces recherches, assurer la pertinence scientifique, la validité, la fiabilité? L'objet de cet article est bien de formaliser un ensemble de critères cohérents et incontournables pour toute recherche scientifique en éducation et qui sont à examiner selon trois aspects essentiels: la conceptualisation, le recueil et le traitement des données, l'énonciation des conclusions. En examinant quelques questions actuelles au-delà de sectarismes épistémologiques et méthodologiques, l'auteur ne renonce à confirmer la fonction première de toute entreprise scientifique: produire de l'intelligibilité.

DE KETELE Jean-Marie, "La publication scientifique en sciences de l'éducation et de la formation: état des lieux, dangers et perspectives ", Les Dossiers des sciences de l'éducation, 2019, no 41, p. 43-59 [en ligne]

L'auteur s'intéresse à la «fonction de dissémination des connaissances » des sciences de l'éducation et de la formation. Il dresse un état des lieux des formes de publications scientifiques écrites, revues et ouvrages, et montre les évolutions majeures que connaît ce secteur. Des mutations récentes s'observent, dues aux effets de l'évaluation des écrits scientifiques, aux modèles économiques développés et à la politique de l'open access.

https://journals.openedition.org/dse/3320

DOUSSOT Sylvain, ROCHEX Jean-Yves (sous la direction de), "Recherche, politique et pratiques en éducation / 1: services rendus et questions posées d'un univers à l'autre. Dossier ", Revue française de pédagogie, juillet-août-septembre 2017, n² 200, p. 11-97.

Ce numéro s'intéresse d'abord au débat entre chercheurs, experts et politiques dans la mise en place de l'éducation inclusive en République tchèque et aux relations entre politique et recherche dans la refondation de l'éducation prioritaire en France. Il analyse ensuite un 
mouvement socio-pédagogique belge visant à infléchir les politiques éducatives et à améliorer les pratiques enseignantes en faveur de jeunes de milieu populaire ou encore l'usage des outils de la recherche dans l'accompagnement des établissements scolaires suisses.

https://journals.openedition.org/rfp/6885

DURU-BELLAT Marie, "La recherche en éducation, une recherche qui accepte d'être utile? ", in MABILON-BONFILS Béatrice, DELORY-MOMBERGER Christine (sous la direction de), À quoi servent les sciences de l'éducation?, Paris: ESF, 2019, p. 59-68

L'apport potentiel des recherches en éducation est souvent mis en balance avec les spécificités et tensions qui caractérisent ce champ. Et le chercheur en éducation est souvent tiraillé entre le politique et sa demande d'expertise et les acteurs et leurs quêtes de recettes. L'auteure analyse les résistances face au risque d'une approche trop utilitariste et défend des rapports plus serrés entre recherche et action.

GAUSSEL Marie, "Production et valorisation des savoirs scientifiques sur l'éducation", Lyon: Institut français de l'éducation, Dossier de veille de l'IFE, décembre 2014, $n^{\circ}$ 97, 28 p. [en ligne]

Dans un contexte de reddition de comptes, en particulier dans le domaine de la recherche publique, la question de l'utilité des recherches sur l'éducation est de plus en plus abordée. Ce dossier interroge la façon dont les résultats des recherches en éducation sont produits, diffusés et valorisés dans un contexte de mutation du paysage de l'information scientifique, avec en particulier le développement des publications en libre accès. https://bit.ly/3qweNE3

KNAUPP Monika, SCHAUFLER Sarah, HOFBAUER Susann, KEINER Edwin, "Education research and educational psychology in Germany, Italy and the United Kingdom - An analysis of scholarly journals", Revue suisse des sciences de l'éducation = Swiss Journal of Educational Research, 2014, vol. 36, $n^{\circ}$ 1, p. 83-108 [en ligne]

L'article étudie les récentes trajectoires transnationales et transdisciplinaires des savoirs sur la base des revues scientifiques, en se focalisant sur les relations, les points communs et les différences entre la recherche en éducation et la psychologie de l'éducation, cela dans trois pays: l'Allemagne, l'Italie et l'Angleterre. Les auteurs examinent les caractéristiques de la recherche en éducation et de la psychologie de l'éducation concernant les auteurs, les thématiques principales et les approches méthodologiques. Ils identifient différents modèles de recherche par rapport aux nations et cultures, d'une part, et par rapport aux disciplines, d'autre part. https://sjer.ch/article/view/4928/7210

MAGRATH Bronwen, ASLAM Monazza, JOHNSON David, "Systems research in education: Designs and methods", Research in Comparative and International Education, mars 2019, vol. 14, $n^{\circ}$ 19, p. 7-29 [en ligne]

Les auteurs cherchent à éclairer les défis méthodologiques de la recherche sur les systèmes éducatifs. Il existe un consensus croissant sur le fait que les interventions visant à améliorer les résultats d'apprentissage doivent être conçues et étudiées dans un cadre plus large, et que les résultats d'apprentissage sont affectés par un réseau complexe de dynamiques impliquant les acteurs, les processus et les contextes sociopolitiques. Les auteurs s'appuient sur des recherches récentes et en cours dans le cadre du programme de recherche Raising Learning Outcomes in Education Systems. https://bit.ly/2LOmRfZ 


\section{ReCHERCHE et POLITIQUeS ÉDUCATIVES}

CYTERMANN Jean-Richard, "La recherche en éducation et les décideurs: des relations intermittentes, des évolutions à confirmer ", Revue française de pédagogie, janvier-février-mars 2018, $n^{\circ}$ 202, p. 9-17 [en ligne]

En France, la recherche en éducation, à la différence de la recherche en santé, ne joue pas un rôle d'appui aux politiques publiques. Cette absence de visibilité de la recherche en éducation tient à la fois à une offre de recherche peu structurée et à une demande intermittente des décideurs publics. L'article essaie de donner quelques éléments d'explication à la faiblesse de la demande: la recherche en éducation a une image parfois négative auprès des décideurs; ceux-ci ont d'autres sources d'expertise en interne que les travaux de recherche; les décideurs ne savent pas passer des commandes de recherche. Quelques signes d'évolution positive apparaissent cependant dans les années récentes et restent à confirmer sur la longue durée. https://journals.openedition.org/rfp/7414

DOUSSOT Sylvain, ROCHEX Jean-Yves (sous la direction de), "Recherche, politique et pratiques en éducation / 3: la recherche en éducation à l'interface du politique", Revue française de pédagogie, janvier-février-mars 2018, $n^{\circ}$ 202, p. 5-70 [en ligne]

Ce numéro évoque, en France, les relations entre la recherche et les décideurs au sein du Conseil national d'évaluation du système scolaire (Cnesco), qui visait à faire le lien entre recherche, évaluation, pratiques et politiques éducatives ou encore les relations entre la recherche et la politique dans l'enseignement de l'histoire au Québec.

https://journals.openedition.org/rfp/7398

LESSARD Claude, "Recherche et politiques éducatives", in VAN ZANTEN Agnès, RAYOU Patrick (sous la direction de), Dictionnaire de l'éducation, Paris : PUF, 2017 (2e édition), p. 716-722

Lauteur propose une réflexion de type politique et socio-épistémologique sur les politiques éducatives et le rôle joué par la recherche en sciences humaines et sociales dans les universités, les centres de recherche, les organisations internationales, ainsi que les think tanks et fondations.

NORMAND Romuald, Gouverner la réussite scolaire: une arithmétique politique des inégalités, Berne: Peter Lang, 2011, 260 p.

Comparant les États-Unis, l'Angleterre et la France, ce livre éclaire le débat public sur les inégalités à l'école face aux enquêtes internationales utilisées pour légitimer des choix stratégiques et à l'obligation de rendre compte qui s'installe dans les systèmes éducatifs. Science et politique étant liées, l'auteur explore la circulation internationale des savoirs scientifiques et experts, leur traduction en sciences de gouvernement, leur stabilisation dans des recommandations et des instruments choisis par les décideurs politiques pour justifier les réformes. 
REY Olivier, "Quand les politiques éducatives appellent la recherche à la rescousse ", Administration et éducation, septembre 2018, $n^{\circ} 159$, p. 95-100

En France, la recherche est régulièrement convoquée pour jouer un rôle dans la conception et dans le développement des politiques éducatives. Cette relation entre le savoir scientifique et la conduite des politiques publiques en éducation est un mouvement international que l'on constate dans de nombreux pays depuis la fin du $\mathrm{xx}^{\mathrm{e}}$ siècle. Plusieurs réformes aux États-Unis ou en Angleterre, depuis les années 1990, ont été nourries par une abondante littérature plaidant pour une recherche en éducation réorientée vers la production de résultats utilisables par les politiques éducatives.

VAN ZANTEN Agnès (coordinatrice), "Connaissances et politiques d'éducation: quelles interactions? Dossier", Revue française de pédagogie, mars 2013, $n^{\circ} 182$, p. 5-40 [en ligne]

Après l'article introducteur d'Agnès van Zanten, les trois articles du dossier abordent différents aspects de l'interaction entre les connaissances et les politiques éducatives, en se fondant sur des recherches menées dans le cadre du projet de recherche européen Know \& Pol. Mobilisant des courants divers de la science politique et de la sociologie, ils mettent en évidence le rôle central des connaissances dans la conduite des politiques éducatives, mais aussi la façon dont les modèles politiques dominants et les conjonctures politiques influent sur la réception et l'utilisation des connaissances. https://journals.openedition.org/rfp/3977

\section{RECHERCHE ET PRATIQUES ÉDUCATIVES}

$B R U$ Marc, "De quelques reconfigurations du rapport des recherches aux pratiques enseignantes", Les Sciences de l'éducation pour l'ère nouvelle, 2019, vol. 52, $n^{\circ}$ 1, p. 79-101

L'article s'intéresse aux rapports entre les recherches et les pratiques enseignantes au cours des années qui ont suivi la reconnaissance universitaire des sciences de l'éducation en 1967, en France. L'auteur en analyse les principales étapes et identifie les faiblesses du transfert des résultats des recherches sous forme de simple application en termes de bonnes pratiques. Le développement de recherches consacrées à la description, à la compréhension et à l'explication du fonctionnement des pratiques enseignantes dans leurs contextes de réalisation offre la perspective de nouveaux rapports entre les recherches et les pratiques enseignantes.

DOUSSOT Sylvain, ROCHEX Jean-Yves (sous la direction de), "Recherche, politique et pratiques en éducation / 2: espaces, acteurs et supports de médiation ", Revue française de pédagogie, octobre-décembre 2017, no 201, 129 p. [en ligne]

Ce numéro s'intéresse à la place du Conseil supérieur de l'éducation du Québec, un organisme de représentation citoyenne visant à éclairer les politiques publiques en éducation, et à celle du Conseil supérieur des programmes français au sein de l'Éducation nationale. Il évoque aussi les compétences du " passeur » en éducation, le rôle de la recherche pour penser les réformes des administrations scolaires en France, ou encore la construction de séquences didactiques fondées sur la recherche au Mexique. https://journals.openedition.org/rfp/7143 
FARLEY-RIPPLE Elizabeth, MAY Henry, KARPYN Allison et al., "Rethinking connections between research and practice in Education: A conceptual framework", Educational Researcher, mars 2018, vol. 47/4, p. 235-245

Les récents efforts pour améliorer la qualité et l'accessibilité de la recherche scientifique en éducation, liés aux attentes accrues pour l'utilisation de la recherche dans la pratique, appellent de nouvelles façons de penser les liens entre la recherche et la pratique. Le cadre conceptuel présenté dans cet article soutient que l'accroissement de la recherche dans la prise de décision dans l'éducation ne peut pas être réduit à une question de diffusion ou de motivation des professionnels de l'éducation à accéder à la recherche fondée sur des preuves. Il s'agit plutôt de renforcer les liens entre la recherche et la pratique dans l'éducation.

GAUSSEL Marie, "Les pratiques enseignantes face aux recherches", Lyon: Institut français de l'éducation, Dossier de veille de l'IFÉ, février 2020, 36 p. [en ligne]

La volonté d'adosser les politiques et les pratiques éducatives aux résultats de la recherche s'amplifie depuis plusieurs années, en France, comme à l'étranger. Afin de mieux prendre la mesure des enjeux concernant l'utilisation des recherches par les enseignants, ce dossier porte d'abord sur la nature des recherches concernées et leur vocation à guider les décisions politiques. Il questionne ensuite le principe d'adossement à la recherche dans le cadre de la formation initiale et sa validité face aux savoirs d'expérience développés ensuite par les enseignants et enseignantes. En dernier lieu, l'auteure pose la question des conditions de mobilisation des résultats de recherches par le corps enseignant. https://bit.ly/3osDHmk

HATTIE John, YATES Gregory, L'apprentissage visible: ce que la science sait de l'apprentissage, Breuillet: Éditions l'Instant présent, 2020 (édition originale 2014), $417 p$.

L'ouvrage de John Hattie, chercheur en éducation néozélandais, et de Gregory Yates, chercheur en psychologie australien, est entièrement consacré au concept d'apprentissage. Il identifie les principaux aspects de la vie à l'école pour conduire à un apprentissage maximisé des élèves. Les auteurs ont passé en revue des milliers d'études concernant les recherches en pédagogie, en psychologie de l'éducation et en sciences cognitives pour en extraire les résultats sur plusieurs thèmes parmi lesquels la relation enseignant-élève, le retour d'information, l'enseignement explicite, la confiance en soi ou les mécanismes de l'apprentissage et de la mémorisation.

MARION Caroline, HOULFORT Nathalie, "Transfert de connaissances issues de la recherche en éducation: situation globale, défis et perspectives ", Nouveaux Cahiers de la recherche en éducation, 2015, vol. 18, no 2, p. 56-89 [en ligne]

Le transfert des connaissances issues de la recherche s'impose comme un champ d'investigation incontournable dans plusieurs domaines. Tant en santé, en sciences sociales, humaines et appliquées qu'en éducation ou en gestion, des recherches visent à réduire le fossé entre les connaissances produites et ce qui est observé dans la pratique. Découlant d'une recension des écrits publiés entre 2007 et 2013, l'article propose de situer le transfert des connaissances en éducation au regard de ce qui se passe globalement dans les autres domaines. Il en aborde les distinctions ainsi que les défis et les perspectives. https://bit.ly/3LLYdNO 


\section{PENUEL William R., GALLAGHER Daniel J., Creating Research-Practice} Partnerships in Education, Cambridge, MA : Harvard education press, 2017, 256 p.

Les auteurs présentent les objectifs des partenariats recherche-pratique en éducation qui cherchent à développer des collaborations à long terme dans lesquelles les éducateurs et les chercheurs travaillent ensemble pour étudier et résoudre des problèmes de pratique urgents. En s'appuyant sur un large éventail d'exemples, ils décrivent les formes que ces efforts peuvent prendre, les défis auxquels ils sont généralement confrontés et les stratégies pour surmonter les difficultés. Le livre comprend des outils pour travailler ensemble ainsi que des stratégies pour assurer l'équité de la participation des deux côtés.

PERRENOUD Philippe, "Recherche et politiques éducatives", in VAN ZANTEN Agnès, RAYOU Patrick (sous la direction de), Dictionnaire de l'éducation, Paris : PUF, 2017 (2édition), p. 722-727

Pourquoi la recherche en éducation a-t-elle si peu d'effets sur les pratiques pédagogiques? Comment rendre l'enseignement plus efficace? L'auteur considère que les enseignants sont en quête de savoirs utiles à la réussite de leur action. Il faut mettre les savoirs issus de la recherche au service de l'intelligence au travail et préparer les enseignants à trouver euxmêmes des réponses qui leur conviennent.

\section{LES DÉBATS RÉCENTS EN SCIENCES DE L’ÉDUCATION}

BRYK Anthony S., ENGESTRÖM Yrjö, MEURET Denis et al., "Pratiques fondées sur la preuve, preuves fondées sur la pratique? Dossier ", Éducation et didactique, novembre 2017, vol. 11, $n^{\circ}$ 2, p. 9-61 [en ligne]

Ce dossier est né de la rencontre d'un texte d'Anthony S. Bryk, président de la Carnegie Foundation for the Advancement of Teaching, à l'université Stanford (Californie) et de diverses contributions ou commentaires de chercheurs et chercheuses en éducation de différentes origines et sensibilités. L'article de A. S. Bryk utilise deux syntagmes, repris à la théorisation récente de la clinique médicale: celui de "pratiques fondées sur la preuve " (evidence-based practice) et celui de "preuves fondées sur la pratique»(practice-based evidence). https://journals.openedition.org/educationdidactique/2716

DEHAENE Stanislas (sous la direction de), La science au service de l'école: premiers travaux du Conseil scientifique de l'éducation nationale, Paris: Odile Jacob, 2019, $286 p$.

La recherche sur les mécanismes cognitifs et cérébraux des apprentissages a produit des résultats majeurs ces vingt dernières années. L'ouvrage présente les premiers travaux du Conseil scientifique de l'éducation nationale en France. Il apporte un éclairage scientifique sur les enjeux éducatifs actuels. Comment réduire les inégalités scolaires en détectant les besoins des enfants et en y répondant dès la première année d'école? Comment enseigner la lecture et la compréhension des mots et des textes? Comment motiver les enfants et faire croître leur envie d'apprendre? 
EMPLIT Philippe, ZHANG Thérèse (coordinateurs), Evidence-based approaches to learning and teaching: Thematic Peer Group Report, Brussels: EUA : European university Association, mars 2020, 13 p. [en ligne]

Ce rapport est le résultat de travaux menés par un groupe de chercheurs autour du concept anglo-saxon d' "evidence-based approaches to learning and teaching" ou "approches de l'apprentissage et de l'enseignement par la preuve ". L'objectif est de chercher comment améliorer l'apprentissage et l'enseignement à partir d'une méthodologie qui repose sur des données probantes, concrètes, et expérimentées en cours. Les auteurs montrent comment cette approche pourrait être adoptée à grande échelle et développée plus avant dans les établissements d'enseignement supérieur à travers l'Europe. https://journals.openedition.org/questionsvives/1170

\section{GUERRIERO Sonia (ed.), Pedagogical Knowledge and the Changing Nature of the} Teaching Profession, Paris: OCDE, février 2017, 276 p. [en ligne]

Les enseignants sont confrontés actuellement à des attentes plus élevées et plus complexes pour amener les élèves à atteindre leur plein potentiel. La nature et la variété de ces exigences impliquent que les enseignants soient des professionnels qui prennent des décisions basées sur une base de connaissances solide et actualisée. Le rapport présente des recherches et des idées à partir de multiples perspectives sur les connaissances pédagogiques. Il examine la dynamique des connaissances dans la profession enseignante et étudie comment les connaissances des enseignants peuvent être mesurées. https://bit.ly/3gcUWoC

HOUDE Olivier, BORST Grégoire, ANDRÉ Christophe et al. (coordinateurs), Le cerveau et les apprentissages, Paris: Nathan, 2018, 335 p.

À travers les analyses de trente experts de différentes disciplines, l'ouvrage s'intéresse aux neurosciences cognitives, aux interactions entre le cerveau et les apprentissages (lire, écrire, compter et penser) et aux fonctions cognitives transversales du cerveau (l'attention, la mémoire, l'auto-évaluation et la métacognition...). Il donne aussi des exemples d'application en neurosciences et en sciences cognitives (sciences et éducation, troubles de l'apprentissage et difficultés scolaires, formation des enseignants...).

\section{LENA Pierre, AJCHENBAUM-BOFFETY Béatrice (coordinateurs), Éducation,} sciences cognitives et neurosciences: quelques réflexions sur l'acte d'apprendre, Paris: PUF, 2008, $226 p$.

Depuis la décennie 1990, les neurosciences cognitives et la compréhension du cerveau connaissent une expansion considérable et éclairent les processus d'apprentissage. L'Académie des sciences, en collaboration avec le Centre pour la recherche et l'innovation dans l'enseignement (Ceri) de l'OCDE, a organisé, en novembre 2005, un séminaire sur le thème "Éducation, cognition et cerveau ", associant des spécialistes de neurosciences. Que nous apprend l'investigation directe du cerveau? Quels éclairages apporte-t-elle sur l'acte d'apprendre, qu'il s'agisse de la lecture, des langues, de la mémoire ou du calcul?

MAROY Christian (sous la direction de), "Réguler les systèmes scolaires par les connaissances: instruments, usages, effets. Dossier", Recherches sociologiques et anthropologiques, 2012, $n^{\circ}$ 43-2, 119 p. [en ligne]

Le dossier a pour visée générale d'interroger la genèse, les objectifs, les significations variées, les usages nationaux ou locaux diversifiés, les effets politiques, cognitifs et pragmatiques 
de la mise en place des instruments basés sur les connaissances visant à "gouverner par les résultats » et/ou "par l'apprentissage » dans le domaine de l'éducation. Il interroge également les modes de construction de ces instruments, leurs modalités de mise en ouvre, ainsi que les controverses et tensions qu'elles suscitent. https://journals.openedition.org/rsa/780

MOLINA Ezequiel, PUSHPARATNAM Adelle, RIMM-KAUFMAN Sara et al., Evidence-Based Teaching: Effective Teaching Practices in Primary School Classrooms, Washington: Banque mondiale, novembre 2018, 63 p. [en ligne]

Dans les pays à revenus faibles ou intermédiaires, une part importante des élèves termine l'école primaire sans avoir acquis les compétences de base en lecture, écriture et mathématiques. Un nombre croissant de recherches suggèrent que cette crise de l'apprentissage reflète une crise de l'enseignement. Que peuvent faire les enseignants? Les auteurs présentent une synthèse des pratiques de classe efficaces et fondées sur les preuves dans l'enseignement primaire. Ils exposent les fondements théoriques du nouvel outil d'observation en classe de la Banque mondiale, Teach. https://bit.ly/37B9Px7

NORMAND Romuald, "Les qualités de la recherche ou les enjeux du travail de la preuve en éducation", Éducation et sociétés, février 2006, n 18, p. 73-91 [en ligne sur le portail Cairn]

À l'échelle internationale, la recherche en éducation fait l'objet d'une tentative de normalisation dans ses méthodes et ses résultats au nom de l'amélioration de la qualité de l'enseignement et de la diffusion de bonnes pratiques. L'article interroge ces présupposés normatifs en les resituant dans leur conjoncture politique et en présentant les débats suscités aux États-Unis et en Grande-Bretagne dans le monde de l'éducation. Il met aussi en question la pertinence d'un «modèle de l'ingénieur " qui, se focalisant sur les applications pratiques et immédiates des résultats de recherche, sous-estime le bien-fondé des principes et des logiques d'action au sein d'une communauté épistémique.

QUÉRÉ Olivier, "De la recherche en éducation aux pratiques éducatives: une comparaison internationale des usages de l'Evidence-based education ", Revue internationale d'éducation de Sèvres, avril 2017, $n^{\circ}$ 74, p. 24-29 [en ligne]

Depuis le milieu des années 2000, des chercheurs et experts recourent au concept d'evidence-based policy (la politique informée par la recherche scientifique) pour qualifier les pratiques de transfert de la science vers la politique. Certains chercheurs des sciences de l'éducation ont recours au concept d'evidence-based education pour apporter au domaine de l'éducation les garanties d'une scientificité et d'une utilité sociale. L'auteur propose un tour d'horizon des pratiques de knowledge brokering en Europe, qui contribue à structurer un certain type de politiques et de pratiques. Il étudie deux modèles différents de dispositifs de transferts de savoir: la « dissémination » et l'experience-based education.

https://journals.openedition.org/ries/5770

SENSEVY Gérard, SANTINI Jérôme, CARIOU Didier, QUILIO Serge, "Preuves fondées sur la pratique, pratiques fondées sur la preuve: distinction et mise en synergie ", Éducation et didactique, 2018, vol. 12, $n^{\circ} 2$, p. 111-125 [en ligne]

L'article s'inscrit dans la suite des travaux de A. S. Bryk et de son usage de l'idée de preuve fondée sur la pratique (practice-based evidence), notion d'abord proposée en médecine. Les chercheurs qui l'ont élaborée l'ont fait à la fois en réaction et en complément à une recherche 
attachée à déterminer des "pratiques fondées sur les preuves » (evidence-based practice), qui sont d'ordre statistique. Les auteurs proposent une lecture de l'article de A.S. Bryk en précisant ce que peut signifier cette expression et en définissant dans quelle épistémologie de la pratique elle s'inscrit. Ils s'attachent ensuite aux conséquences de cette conception sur les relations entre la pratique et la recherche. Enfin, ils insistent sur la nécessité de mise en synergie des preuves anthropologiques et des preuves statistiques.

https://journals.openedition.org/educationdidactique/3400

TARDIF Éric, BLANCHETTE Jérémie, MASSON Steve et al., "La psychologie cognitive s'invite en classe ", Enjeux pédagogiques, 2017, $n^{\circ} 28, p .12-41$ [en ligne]

Les résultats des études scientifiques menées dans les champs des neurosciences et de la psychologie cognitive ont parfois abouti à des représentations et des réflexions simplifiées, voire déformées, quant à la contribution des neurosciences dans les processus d'enseignement et d'apprentissage. Ce numéro a pour objectif d'approfondir la thématique de la psychologie cognitive en classe et d'apporter une vision différente de cette contribution des neurosciences. De nombreux exemples à propos de la mémoire, du raisonnement, des mathématiques, de l'orthographe et de la lecture permettent d'illustrer l'apport de la psychologie cognitive pour les enseignants et les élèves, à travers différents témoignages.

https://bit.ly/3osESSM

\section{TARDIF Éric, DOUDIN Pierre-André (sous la direction de), Neurosciences et} cognition: perspectives pour les sciences de l'éducation, Louvain-la-Neuve: De Boeck Supérieur, 2016, 412 p.

164 L'ouvrage fait le point sur les débats actuels entre la pertinence d'une collaboration entre les neurosciences cognitives et les sciences de l'éducation en ce qui concerne notamment le langage, la mémoire, l'attention, le raisonnement, l'apprentissage et les troubles qui lui sont reliés. Les auteurs (canadiens, américains, français, suisses) donnent un aperçu général des résultats de recherches récentes et font le point sur de nouvelles avancées en neurosciences cognitives en lien avec les sciences de l'éducation. Bien que prometteuse, cette collaboration entre neurosciences, sciences cognitives et sciences de l'éducation comporte plusieurs obstacles (attentes irréalistes, interprétations abusives de résultats de recherche, fausses croyances...).

TOSCANI Pascale, CONNAC Sylvain (coordinateurs), "Neurosciences et éducation ", Éducation et socialisation. Les Cahiers du CERFEE, septembre 2018, $n^{\circ} 49$, n.p. [en ligne]

À la suite du rapport de l'OCDE (2007) Comprendre le cerveau: naissance d'une science de l'apprentissage, les neurosciences ont fait leur entrée dans le domaine de l'éducation scolaire et de la formation. Parallèlement, les résultats de programmes internationaux ont conduit les neuroscientifiques à remettre en cause certains concepts liés à l'apprentissage. Cette entrée des neurosciences dans le monde de l'éducation a provoqué une rupture épistémologique, qui aujourd'hui encore prête à débat. Ce dossier vise à clarifier les rapports que les neurosciences entretiennent avec le monde de l'éducation. https://journals.openedition.org/edso/3091 